\title{
LA POÉTICA DE LA TRADUCCIÓN DE THOMAS MERTON EN SUS CARTAS A ESCRITORES
}

\author{
Marcela María Raggio* \\ Universidad Nacional de Cuyo \& CONICET/ Argentina
}

\begin{abstract}
Resumen: Este artículo busca glosar la poética de la traducción de Thomas Merton a partir del estudio de sus cartas a escritores. En ellas, Merton reflexiona acerca de lo que significa la traducción poética, los modos de traducir, y la experiencia de acercarse a lo extranjero (el extranjero) mediante la traducción. Luego, se realiza un análisis traductológico de una muestra de traducción por Merton, a fin de revisar la conexión entre su poética y su práctica de la traducción.
\end{abstract}

Palabras clave: Thomas Merton. Poesía latinoamericana. Traducción.

\section{THOMAS MERTON'S POETICS OF TRANSLATION IN HIS LETTERS TO WRITERS}

\begin{abstract}
This article explores Thomas Merton's poetics of translation as reflected in his letters to writers. There, Merton expresses his ideas on poetic translation, the methods and the experience of approaching foreign literature through translation. Then, a translation analysis of a sample revises the connection between Merton's poetics and practice of translation. Keywords: Thomas Merton. Latin American poetry. Translation.
\end{abstract}

\footnotetext{
* Licenciada en Letras y Filología Inglesa, y Magister en Literatura Hispanoamericana por la UNCuyo (Argentina). Máster en Historia y Estética del Cine por la Universidad de Valladolid (España). Doctora en Letras por la UNCuyo. Actualmente, Profesora Titular Efectiva de Literatura Británica y Literatura Norteamericana en la Universidad Nacional de Cuyo (Mendoza, Argentina) e Investigadora del Consejo Nacional de Investigaciones Científicas y Técnicas de Argentina (CONICET). Mendoza, Argentina. E-mail: marcelaraggioo@yahoo.com.ar
} 


\section{Introducción}

Las traducciones poéticas realizadas por Thomas Merton conforman una parte importante de su obra. Las de poetas latinoamericanos fueron incluidas, en gran medida, en el volumen Emblems of a Season of Fury (1961); aunque también deben considerarse la publicación póstuma de The Jaguar and the Moon - El jaguar y la luna (de Pablo A. Cuadra) en 1974; y los Poems and Anti-Poems de Nicanor Parra, en 1967.

En general, podría presuponerse que la traducción poética (tal como la practican los escritores/poetas) es un mero ejercicio para ciertos escritores (aunque el hecho de que salgan de sí mismos, de su obra, y se adentren en la de otros autores, en otras lenguas, conlleva un interés por el Otro). La obra traducida puede servir como inspiración, como práctica, como búsqueda de nuevos caminos para la poesía propia. Pero la actividad de traducción tiene, además, un componente ético que en el caso de la traducción poética pasa (o debería pasar) al primer plano. La traducción pone al poeta-traductor frente a una disyuntiva: o bien adapta lo desconocido a lo conocido y "domestica" el texto; o bien muestra en su versión la "extranjeridad" del Otro, intentando comprenderlo en su alteridad. Esta segunda opción es la que Antoine Berman propone como alternativa ética de la traducción poética.

La tarea de traducción de Thomas Merton debe ser enmarcada en esa opción por entender al Otro, ubicarlo en su lugar propio y hacer que otros también lo vean así. Planteamos esta hipótesis particularmente para la poesía latinoamericana que tradujo Merton. ${ }^{1}$ La elección de los poetas, la relación epistolar que mantuvo con varios de ellos, su apreciación crítica de la poesía latinoamericana y las versiones resultantes pueden ser estudiados teniendo en cuenta dos parámetros: por un lado, el posicionamiento de Merton ${ }^{1}$

\footnotetext{
${ }^{1}$ Sus traducciones del chino, persa, griego y latín pueden haberse originado en otros motivos; y lo mismo puede decirse de las traducciones del francés. Ya que esto escapa a nuestro análisis, nos centramos en la poesía latinoamericana exclusivamente.
} 
como poeta americano y, por otro, lo que ponen de manifiesto los textos traducidos. Es decir, la selección de Merton se relaciona no solamente con su preferencia personal, su amistad con ciertos poetas, o la difusión de estos en sus países de origen; sino que, sobre todo, está dada por la postura crítica que manifiestan los textos frente a la realidad latinoamericana, postura que es compartida por Merton.

En La traduction et la lettre, Berman sostiene: "Telle est la traduction: expérience." (1999, 16) La experiencia de las obras, de la lengua del original, de la lengua de llegada, y la reflexión sobre esa experiencia, dan lugar a lo que Berman llama la "traductología": "la réflexion de la traduction sur elle-même á partir de sa nature d'experiénce." (17) Las dos nociones sobre las que construye Berman su teoría de la traducción, experiencia y reflexión, resultan fundamentales para comprender la poética de la traducción de Merton. La traductología, sostiene Berman, es “une pensée-de-la-traduction." (19)

Asimismo, si se considera el profundo interés que Merton expresa por las obras literarias latinoamericanas que incorpora a sus libros de autor, las siguientes palabras de Berman resultan sumamente claras: “... les oeuvres elles-mêmes, à l'âge moderne, se conçoivent comme traduction." (19) De este modo, la obra de Merton podría inscribirse en la línea de escritores y pensadores que señala Berman: Hamann, Proust, Valéry, Roa Bastos, Pasternak, Marina Tsvétaïeva et al. para quienes "la traduction signifie non seleument le 'passage' interlangues d'un texte, mais ... toute une série d'autres 'passages' qui concernent l'acte d'écrire et, plus secrètement encore, l'acte de vivre et de mourir." (21) La idea central de Antoine Berman acerca de la multiplicidad posible de traducciones resulta en la consideración de la traducción como experiencia : la "expérience qu' est la traduction dans son essence plurielle" (23).

En este sentido, resulta relevante un aporte de Henri Meschonnic, otro de los pensadores de la traducción que plantean la noción de ética en el campo que nos ocupa. Para Meschonnic, la traducción y los estudios sobre traducción deben tomar conciencia 
de la historicidad de las versiones. Meschonnic sostiene que "une traduction est une acte de langage... Elle a sa propre historicité." (18) De ahí que considere que la crítica de la traducción (que es un trabajo eminentemente reflexivo), sea "un travail de palimpseste" (19) en el que entran en juego el texto en la lengua A, las diferentes versiones que existen, históricamente, en la lengua $\mathrm{B}$, o en otras, y los contactos culturales que ponen de manifiesto la transtextualidad propia de toda traducción. "Les transformations d'une traduction à l'autre d'un même texte, à la fois transformations de la traduction et transformations du texte" (175) muestran la reciprocidad entre lectura del texto, traducción, y la historicidad de ambas. Muchos de los poemas traducidos por Merton habían sido volcados al inglés antes por los propios autores (por ejemplo, por Ernesto Cardenal); y existían intentos de dar a conocer esos textos en publicaciones angloamericanas. Las reflexiones de Merton en sus cartas a escritores giran en torno de estos tres componentes (lectura, traducción e historicidad), de donde resulta una consideración dialógica de la traducción: las versiones de Merton dialogan con la obra original y con otras versiones preexistentes.

\section{La traducción como posicionamiento}

En muchos de sus escritos, Merton manifiesta sentimientos de admiración, respeto y nostalgia por Latinoamérica. Desde el claustro sueña con poder formar parte de una fundación monástica en Chile $^{2}$; desea respirar el aire de la selva amazónica ${ }^{3}$; se solidariza con los movimientos revolucionarios de diferentes países ${ }^{4}$; y en

\footnotetext{
${ }^{2}$ Hay ciertas alusiones a esta fundación en cartas dirigidas a E. Cardenal durante el año 1966.

${ }^{3}$ En una carta que escribe al argentino Alejandro Vignati, cuando este se encuentra en Brasil (Merton, 1993, 234).

${ }^{4}$ Entre las cartas más evidentes en este sentido se cuentan las que dirige a los poetas nicaragüenses.
} 
todos los casos, pone en palabras las ideas que manifestara en su "Carta a Pablo A. Cuadra acerca de los gigantes":

The largest, richest and best developed single land-mass South of the Equator is South America. The vast majority of its population is Indian, or of mixed Indian blood. [...] The deepest springs of vitality in these races have been sealed up by the Conqueror and Colonizer [...] But if this stone is removed from the spring perhaps its waters will purify themselves by new life and regain their creative, fructifying power. (Merton, Emblems, 1961,77-78)

Ese futuro que Merton prevé en Latinoamérica va tomando forma a través de la poesía. La mirada crítica de Merton sobre la obra de los poetas latinoamericanos establece conexiones entre los textos y la realidad circundante. Tal relación con la realidad es lo que atrae a Merton y lo lleva no solo a apreciar a sus pares sudamericanos sino, sobre todo, a ubicarse entre ellos. En una carta a Stefan Baciu, fechada el 21 de mayo de 1965, Merton explica:

I feel myself clearly much more in sympathy with the Latin American poets today than with those of North America. I feel that though I write in English, my idiom (poetic idiom at least) is much more that of Latin America than that of the United States. To begin with I feel that the academic poets of the U.S. are simply caught in the most sterile impasse where they do nothing except play esoteric tricks with language. [...] Whereas the Latin American poets seem to me to be alive, to have something honest to say, to be sincerely concerned with life and with humanity. There is some genuine hope left in them, or when they are bitter the bitterness has a maturity and content which make it respectable, and in any case I tend to share it in some ways. (Merton, Courage, 241) 
Esta misma alusión al hecho de sentirse más cercano a los latinoamericanos que a los norteamericanos reaparece en cartas a varios otros escritores (por ejemplo, a Napolean Chow, Hernán Lavín Cerda y Clayton Eshelman, entre otros); y la justificación es siempre la misma: la poesía de Sudamérica tiene un aliento vital que la eleva por sobre la norteamericana; y esa fuerza le viene de su conexión con la realidad social y política de los países del sur del continente. Puede proponerse que para Merton la traducción es un sinónimo de apertura. James Laughlin, quien fiera su amigo, editor de las obras de Merton, nexo con el mundo exterior al monasterio, confidente literario, entre otros roles, sostiene:

He had always been so open to spiritual forces of all kinds. In his readings, as you read the journals and other books, you see this constant openness to other traditions. (Wilkes, 30)

En otros contextos, la apertura de Merton a diferentes tradiciones puede entenderse como su llegada a las religiones y filosofías orientales, a la literatura clásica, etc. Pero en el tema que nos ocupa en estas páginas, la apertura a otras tradiciones se pone de manifiesto en el interés en la literatura hispanoamericana, un interés arraigado asimismo en otro cercano, aquel por las culturas de los pueblos originarios. A partir de la lectura y el conocimiento de sus pares latinoamericanos, de su poesía y de las tradiciones de sus países de origen, Merton se siente movido a traducir para compartir con otros angloparlantes aquello que a él lo deslumbra, y que se halla "al sur del Ecuador", y en una lengua inaccesible para muchos norteamericanos. De las cartas a escritores (a los que traduce, o a otros) se desprende una poética de la traducción en la que se aúnan la reflexión y la práctica. La reflexión se da tanto sobre la obra del Otro como sobre la propia versión, la propia práctica poética. 


\section{Glosa de la poética de la traducción mertoniana}

Merton escribe acerca de una autora a quien no llegó a traducir (Susana Soca); pero paradójicamente, de sus palabras sobre ella surge lo que tal vez sea uno de los aspectos más interesantes de su poética de la traducción: "I have never yet had a moment to translate some of her poems, and I would not want to do this in a rush. Precisely, it would have to be a new creation emerging from communion in the same silence." (Merton, Courage, 166) La traducción como comunión, común-unión, diálogo que, también paradójicamente, se da en el silencio. Comunión en silencio, parecen ser los dos pilares de la poética de la traducción mertoniana. A partir de esa comunión, se entabla un diálogo con el texto mismo yo una reflexión sobre la propia práctica.

Al estudiar la obra de Merton como traductor hay que considerar sus motivaciones. Merton no es un traductor "profesional"; su figura puede ser en cambio entendida como la de un poetatraductor: la elección de autores y textos para verter al inglés se origina en sus preferencias personales, en la admiración que siente por los poetas que lee en otros idiomas, e inseparable de estas razones, en su deseo por difundir las ideas (y en el caso de los latinoamericanos, la conexión entre las ideas y la realidad) que advierte en la poesía ajena. El poeta-traductor argentino Ricardo Herrera sostiene: "La traducción no solicitada [...] no obedece tanto al deseo de dar a conocer un autor, como a la necesidad de hacer nuestro el don verbal de una sensibilidad afín que [...] nos ofrece algo de lo que en ese momento carecemos: fuerza emotiva, palabras vírgenes, nuevos recursos expresivos." (43) La reflexión parece explicar las motivaciones del propio Merton: no es que él mismo carezca de fuerza emotiva, recursos válidos; sino que considera, como deja entrever en sus cartas, que el idioma inglés no es tan apropiado para la expresión poética como el español. Y en ese intento por hacer su poesía más "latinoamericana", al mismo tiempo acerca a los poetas que hablan español, y los traduce. En búsqueda de esos ritmos, expresiones, y fuerza 
emotiva. Es el propio Merton quien señala esa "sensibilidad afín" que halla en la poesía de Latinoamérica.

Por otro lado, la poética de la traducción mertoniana se hace explícita no solo en las reflexiones sobre la poesía extranjera que traduce, sino también en sus comentarios acerca de la poesía propia que otros (por ejemplo, Cardenal) traducen al español. En una carta al nicaragüense, fechada el 11 de marzo de 1961, Merton le dice:

I have received all the copies of the Poemas [Spanish translation of Selected Poems]... / Personally I want to say once again that I think your translation of the poems was a magnificent and truly creative job. It is seldom that a poet is so fortunate in his translators. Reading the poems again I am once again struck by the fact that they have a life of their own in Spanish, almost as though they were destined to be in Spanish as well as in English, by a kind of nativity or natura within themselves. In any case you have found that natura and given it expression. (Merton, 1993, 123)

Un primer aspecto de la poética de la traducción mertoniana remite a la idea de traducción como creación. No obstante, no se trata de una creación ex nihilo, sino que la versión en la lengua extranjera se encuentra presente, en forma embrionaria, en el poema original. La tarea del traductor consiste en encontrar esa semilla y hacerla crecer, darle expresión, hacer hablar al poema en otra lengua.

Las versiones que produce Merton, a su vez, no son definitivas; sino que requieren de revisión. Este aspecto puede ser considerado también parte de su poética de la traducción. Las correcciones y segundas lecturas, por otra parte, pueden provenir de sus colegas hispanoamericanos, tal como pide a Cardenal en una carta sin fecha, de 1962: "Here are the translations of Alfonso [Cortés] that I have done [...] I hope you will point out any errors \& make any suggestions that occur to you. And let me know if you can use more copies." (Merton, 1993,135) De hecho, en la carta siguiente 
a esta, el 17 de noviembre de 1962, Merton agradece a Cardenal las correcciones y le comenta que las ha incluido en las versiones de los poemas de Cortés. (Merton, Courage, 136)

Cardenal pareciera ser uno de los interlocutores más válidos acerca de la traducción poética. Es que con él Merton encontró no solamente un espíritu con quien congeniaba y poseía proyectos comunes, sino además una fuente de conocimiento sobre la poesía latinoamericana y sobre las culturas de los pueblos del sur del continente. El hecho de que ambos hubieran estudiado en Columbia, sufrido un proceso de conversión religiosa y entrado a Getsemaní, siendo poetas además de religiosos, contribuyó en gran medida a que la amistad que se forjara cuando eran maestro y novicio respectivamente continuara luego de que Cardenal abandonara el monasterio. En las cartas que intercambian se pueden leer los nombres de autores, títulos de libros y revistas que Cardenal envía a Merton, y el interés que esto despierta en el monje. Como efecto de ese interés, Merton traduce, entre otros, a los latinoamericanos, aunque no siempre es tarea fácil. En carta del 8 de abril de 1963, agradece a Cardenal el envío de poemas de Cortés y aclara: "They seem to me to be among his best, and not the easiest to translate either." (Merton, Courage, 139). Las cualidades que conforman la excelencia de la poesía de Cortés, su carácter metafísico, que Merton indica en la presentación del poeta (Merton, Emblems, 1993, 141) pueden ser las que, al mismo tiempo, dificulten su traducción. Como parte de su poética de la traducción, entonces, puede considerarse que a mayor densidad de significación, Merton propone una proporcional dificultad de verter el texto a otra lengua.

Otro aspecto de la traducción al que Merton dedica atención e importancia es el referido a la especificidad del vocabulario. Refiriéndose a una versión de Platform Sutra, de Hui Neng, le dice a Cardenal: "[...] unfortunately the translation, though probably good in its own right, uses terminology that misses the real Zen meaning and does not correspond to the kind of language used by the best Zen men, like Suzuki." (Merton, Courage, 1993, Courage 141).Si en este caso se trata de una afirmación sobre un 
texto no traducido por él, hay otras cartas en las que vuelve sobre esta preocupación por las palabras, en textos propios vertidos al español, o de latinoamericanos que él traduce al inglés. Así, le escribe a Ludovico Silva el 30 de junio de 1965: "Properly, the best translation for 'Stranger' in the essay ["Day of a Stranger"] would be extranjero but the idea of exile, of displaced person, of alien, also comes in. How about ajeno?" (226)

De cualquier modo, lo que Merton intenta hacer en sus traducciones de poetas latinoamericanos es, justamente, establecer un diálogo con esos "extranjeros" para que no resulten tan "ajenos." Tal como señala Poks (2007), "art overcomes the subjectobject opposition and culminates in the epiphany of the other / the Altogether Other." (252) La traducción, para Merton, es un camino para revelar al Otro, a los Otros latinoamericanos, a fin de que algún día se recupere la unidad perdida de las Américas. Tal como hemos propuesto en otro artículo ${ }^{5}$, no consideramos que Merton fuera un abogado del panamericanismo (como sostienen la mayoría de los estudiosos de su obra). En todo caso, lo que Merton intenta es que las dos Américas se comprendan mutuamente, y que sean una, sí, pero en la diversidad. "Merton's attentive apprenticeship to things Latin American was part of his ongoing search for wisdom beyond splits and divisions [...]" (Poks 2007, 259)

\section{Reflexión y práctica de la traducción: los poemas de César Vallejo}

En su trabajo sobre Merton y América Latina, Poks analiza las implicancias universales de la poesía de Vallejo, aquellas en las cuales Merton encuentra la resonancia de voces de las que habla la autora polaca. En estas páginas, lo que buscamos es precisar

\footnotetext{
5 Raggio, Marcela. “Thomas Merton's Americanism: A Study of His ideas on America in His Letters to Writers". Revista de Estudios Norteamericanos vol 20, Universidad de Sevilla: 2016.
} 
la reflexión que Merton realiza sobre Vallejo, y proponer una aproximación a su práctica de la traducción.

En una carta de 1965 a Stefan Baciu, quien le había solicitado que especificara sus ideas sobre la literatura y los escritores de Latinoamérica, Vallejo es el único peruano incluido. Sobre él, dice Merton: "I have done a few poems of the great [César] Vallejo whom I consider the most important voice in American poetrry in the twentieth century, in fact probably the greatest poet of the century in any language." (Merton, Courage, 242)

El otro corresponsal con quien Merton intercambia ideas sobre Vallejo es Clayton Eshelman, quien por su parte, también tradujo a Vallejo. De su reflexión sobre Vallejo, surge algún punto más de la poética mertoniana de la traducción. Tras afirmar que ha traducido unos pocos poemas del peruano, y animar a Eshelman en esa tarea, sostiene que "all the poets in America could translate Vallejo and not begin to get him." (Courage 254). De esto se desprende que, para Merton, la traducción es un camino hacia la comprensión del otro; pero el camino es arduo, y nada garantiza que se logre el objetivo. En cierto modo, estas ideas entroncan con aquella que Merton expresaba acerca de Susana de Soca: sólo de la comunión en el mismo silencio surgirá la traducción y, al mismo tiempo, la comprensión. En la misma carta, Merton destaca la universalidad de Vallejo (adjudicándole en su sentido etimológico la palabra "católico") y oponiéndolo a los poetas estadounidenses. Si el propio Merton se sentía lejos de los escritores norteamericanos y se hallaba más a gusto entre los latinoamericanos, en Vallejo encuentra uno de sus pares:

[...] what I mean is that Vallejo is totally human, as opposed to our zombie poets and our little girl poets and our incontinents. [...] he is tremendous and extraordinary, a huge phenomenon [...] Therefore I think that a translation of Vallejo is not only a nice interesting venture but a project of very great and urgent importance for the human race. (Merton, Courage, 1993, Courage 254-255) 
Un último aspecto de la poética de la traducción consiste en que la actividad de los traductores, cuando es verdaderamente relevante, no consiste meramente en un desafío lingüístico, intelectual, de interés, sino en todo caso en un proyecto para la raza humana: la poesía está indisolublemente unida a lo vital, a la supervivencia de la humanidad.

En Emblems..., Merton (1961) traduce cuatro poemas de Vallejo: "Estáis muertos", "Black Stone on Top of a White Stone", "Peace, the Wasp..." y "Anger". Para nuestro análisis nos concentraremos en "Black Stone..." y el poema original, "Piedra negra sobre una piedra blanca." A fin de lograr la reflexión sobre las traducciones, presentamos también los textos originales en español: ${ }^{6}$

"Piedra negra sobre una piedra blanca" (p.205-206)

Me moriré en París con aguacero, un día del cual tengo ya el recuerdo. Me moriré en París - y no me corrotal vez un jueves, como es hoy, de otoño.

Jueves será, porque hoy, jueves, que proso

estos versos, los húmeros me he puesto

a la mala y, jamás como hoy, me he vuelto, con todo mi camino, a verme solo.

César Vallejo ha muerto, le pegaban todos sin que él les haga nada; le daban duro con un palo y duro

también con una soga; son testigos los días jueves y los huesos húmeros, la soledad, la lluvia, los caminos...
"Black Stone on Top of a White Stone" (p.137-138)

I shall die in Paris, on a rainstorm, On a day I already remember.

I shall die in Paris -it does not bother meDoubtless on a Thursday, like today, in autumn.

It shall be a Thursday, because today, Thursday

As I put down these lines, I have set my shoulders

To the evil. Never like today have I turned And headed my whole journey to the ways where I am alone.

César Vallejo is dead. They struck him, All of them, though he did nothing to them. They hit him hard with a stick and hard also

With the end of a rope. Witnesses are: the Thursdays,

The shoulder bones, the loneliness, the rain and the roads...

${ }^{6}$ Tomamos el poema en español de Antología poética, de César Vallejo (Perú, Ed. Chirre, s/f). La versión de Merton está tomada de Emblems of a Season of Fury. 
El poema de Vallejo es un soneto. Los dos cuartetos y los dos tercetos giran en torno a la idea que Poks llama de aceptación sacrificial de Vallejo, de identificación entre el poeta y el Redentor (239). La aceptación, sostiene Poks, no es solamente de la muerte, sino de la condición humana misma; y en su condición escatológica el presente contiene en sí al futuro y trasciende la historia (240). Del mismo modo, afirma Poks, el pasado y el futuro co-forman el presente del poeta (139).

Por nuestro lado, proponemos un análisis que parte desde la forma para adentrarse en el contenido explícito y subyacente del poema. En los dos cuartetos, el poeta conoce el futuro, ya lo está recordando ${ }^{7}$, del mismo modo que recuerda su pasado, signado por la soledad. Si los dos cuartetos están enunciados desde el presente (que subsume pasado y, principalmente, futuro), los dos tercetos modifican la ubicación temporal, y enuncian desde el futuro, pero asumiendo todo el peso del pasado: "César Vallejo ha muerto". Y los testigos de esa muerte son las marcas mismas de la soledad: los días jueves, los huesos húmeros (sobre los que ha llevado su cruz), la lluvia y los caminos, que lo condujeron de los Andes a París.

La versión de Merton (1961) modifica la estructura del poema, al trasladarlo en tres estrofas: las dos primeras, de 4 versos cada una; y los dos tercetos son unificados en una única estrofa de 6 versos. En el primer cuarteto, Merton introduce dos modificaciones significativas. Donde Vallejo dice "Me moriré en París -y no me corro", Merton afirma "I shall die in Paris -it does not bother $m e$ ”. En el poema es español, Vallejo reafirma su decisión de permanecer en París: el exilio forma parte de su ser; y ni siquiera en la hora de su muerte se moverá de la ciudad donde, a pesar de la distancia, encuentra los ecos peruanos que resuenan en su poesía. En la versión en inglés, en cambio, hay una especie de desinterés por la circunstancia. El poeta asume su muerte, y no importa dónde ocurra. El otro cambio se encuentra en el verso siguiente, que

${ }^{7}$ La noción es similar a la que expresa el título de la novela de Elena Garro, Los recuerdos del porvenir. 
Vallejo abre con un "tal vez" que implica posibilidad; mientras que Merton traduce "Doubtless", señalando certeza.

En el segundo cuarteto, además de un cambio en la distribución de la información en los versos (que se debe a la variación en la cantidad de sílabas en español y en inglés), hay un caso de interpretación que se debe, probablemente, al desconocimiento de Merton sobre ciertas expresiones coloquiales en español. Donde Vallejo decía "los húmeros me he puesto huesos a la mala" (indicando dolor, enfermedad o sufrimiento), Merton escribe "My shoulders I have set to the evil." La afirmación, así, adquiere otras connotaciones. Ya no se trata solamente del sufrimiento intrínseco, asociado íntimamente a la soledad - y por qué no, a la humedad del aguacero parisino-, sino que Merton da a los versos una lectura metafísica ${ }^{8}$ Desde nuestra perspectiva, la lectura de Merton resulta valiosa porque pone en juego la idea de que al traducir, vertimos no solo lo que dice el texto original, sino también la lectura que del mismo hacemos, desde nuestro lugar (ontológico, y no solo geográfico). A continuación, Vallejo afirma: "jamás como hoy, me he vuelto, con todo mi camino a verme solo." Merton traduce: "Never like today have I turned I And headed my whole journey to the ways where I am alone." El camino (la vida) forma parte indisoluble del yo en las líneas de Vallejo: el poeta y su camino, el poeta y su vida que lleva a cuestas, giran y se da cuenta de su soledad. Lo acompaña su vida, pero paradójicamente está solo, como ha estado toda su vida. Las palabras con que traduce Merton, en cambio, implican que el poeta gira y reencamina su vida hacia sitios donde está solo. Aquí, el poeta tiene control sobre su vida, que es un ente separado de él, y lo dirige voluntariamente hacia la soledad, en una suerte de elección vocacional hacia la vida monástica. ${ }^{9}$

\footnotetext{
${ }^{8}$ Esta lectura metafísica es evidentemente la que Poks tuvo en cuenta al afirmar que hay en el poema una conexión entre el poeta y Cristo.

${ }^{9}$ Este aspecto es remarcado por Poks en su análisis: la idea, entonces, más que estar presente en el poema de Vallejo, se desprende de la traducción de Merton.
} 
Por último, en los dos tercetos que Merton vierte como un sexteto, hay una adición significativa: los testigos que enumera Vallejo están separados por comas, y los puntos suspensivos con los que concluye el poema señalan la continuidad eterna, la imprecisión temporal y la sugerencia de otros posibles testigos. Merton traduce literalmente, pero agrega la conjunción "and" entre los dos últimos "testigos". Este hecho induce a pensar en una enumeración conclusiva: los testigos son cuatro solamente, y los puntos suspensivos se ven, de este modo, restringidos al eco de las ideas del poema, más que a la posibilidad de que otros hayan observado la muerte del poeta.

Retomando ciertas nociones expresadas más arriba, en el marco teórico, esta versión de un poema de Vallejo por Merton resulta muestra clara del diálogo que establece el traductor con el poeta admirado, de la historicidad de la traducción (ya que hay marcas en el texto que permiten adivinar la interpretación mertoniana monástica del texto de Vallejo) y al mismo tiempo, el hecho de darle lugar al peruano en una obra propia, original, implica el reconocimiento del Otro. La reflexión de Merton acerca de la obra de Vallejo, que se deja entrever en sus cartas, es también la práctica de la escritura para revelar al otro. La lectura de Vallejo, la traducción llevada a cabo, y su ubicación tanto en su canon personal como dentro su propia obra, ponen de manifiesto que para Merton la traducción es una toma de conciencia, un posicionamiento frente a la literatura y a la realidad circundante.

\section{Conclusiones}

"What I mean is that Vallejo is totally human." La aseveración que Merton hace en la carta a Eshelman citada más arriba, indica claramente la necesidad que tenía Merton de traducir, y la postura desde la que lo hacía. Tal como señala en esa misma carta, Merton ve en Vallejo al más humano de los poetas: "he is the most universal, Catholic in that sense (the only real sense), poet of this time [...]" 
(Merton, Courage, 254). Su elección de Vallejo como uno de los grandes poetas de su canon personal, y de su época, y las versiones que de él hace (y que hemos ejemplificado con el análisis de "Piedra negra...") no tienen que ver tanto con el hecho de que Merton halle en Vallejo resonancias religiosas, sino humanas. La soledad que acompaña al peruano en su poema sobre la muerte entrevista, es la soledad de los hombres y mujeres contemporáneos; la que Merton experimenta en el claustro, donde sus proyectos no hallan eco. La postura ética de la traducción en Merton no pasa únicamente por cuestiones lingüísticas, de apego a la forma, o de interpretaciones de contenido: con Vallejo, y con los demás poetas que traduce, Merton intenta establecer un diálogo que tenga eco en sus contemporáneos también, para que se den cuenta, por fin, tal como sostiene en su "Carta a Pablo A. Cuadra, acerca de los gigantes" que, "Latin Americans really existed. That they were real people. That they spoke a different language." (Emblems 85).

\section{Referencias}

Berman, Antoine. La traduction et la lettre, ou l'auberge du lointain. Paris: Éditions du Seuil, 1999.

Herrera, Ricardo. Copia, imitación, manera. Cuadernos de traducción. Buenos Aires: Nuevo Hacer, 1999.

Merton, Thomas. Emblems of a A Season of Fury. New York: Straus, 1961

. The Courage for Truth: The Letters of Thomas Merton to Writers. Edited by Christine M. Bochen, New York: Farrar, 1993. 
Meschonnic, Henri. Poétique du traduire. Normandie: Verdier, 1999.

Poks, Malgorzata.. Thomas Merton and Latin America: A Consonance of Voices. Katowice: Wyższa Szkoła Zarządzania, 2007.

Wilkes, Paul. An Interview with James Laughlin about Thomas Merton. The Merton Annual 26; Conducted by Paul Wilkes, March 18, 1983. Transcribed and edited by Paul M. Pearson, 2013.

Recebido em: 02/01/2016

Aceito em: 28/02/2016 Publicado em maio de 2016 